\title{
Einleitung
}

\subsection{Gegenstand und Hauptthese}

Wissenschaft kommuniziert. Mit diesem Titel möchte ich Wissenschaft nicht, personifizieren': Anstatt in der dritten grammatischen Person des Singulars (er/sie/es kommuniziert), steht kommuniziert im Titel dieser Arbeit für das adjektivisch gebrauchte Partizip Perfekt. So betone ich, dass es sich bei der im Folgenden beschriebenen empirischen Fallstudie, im Ergebnis, um eine Analyse des praktischen Vollzugs von Wissenschaft im Prozess der Kommunikation handelt. Gegenstand meiner Arbeit sind Face-to-face-Kommunikationen zwischen menschlichen Akteuren, die ich für das, was ich hier unter Wissenschaft verstehe, als konstitutiv betrachte (Collins 1998, 25ff). Was für den Alltag aus wissenssoziologischer Perspektive generell gilt, nämlich dass sozial relevant nur das ist bzw. gemacht werden kann, was auch kommuniziert wird (Knoblauch 2013, 27), trifft auch für den Alltag der Wissenschaft zu. Dies gilt umso mehr, wenn im Kontext von Inter- und Transdisziplinarität und jenseits eingespielter Kommunikation kommuniziert wird: Was relevant gemacht werden soll, muss zunächst (erfolgreich) kommuniziert werden, um innerhalb einer Gruppe und über sie hinaus verstanden und geteilt werden zu können. Erst im Ablauf einer gemeinsamen Geschichte wird sich ein, für eine gegebene Gruppe, spezifischer Wissens- und Ausdruckskanon entwickeln und etablieren, der dann retrospektiv als Identitätskern dieser Gruppe betrachtet werden kann.

Aus dieser Tatsache geht ein Umstand hervor, der für die kommunikationssoziologische Perspektive meiner Fallstudie, im Bereich der wissenschaftsinternen interdisziplinären Wissenskommunikation, von größter Bedeutung ist: Kommunikation zielt hier, im Sinne der Relevantmachung von (eigenen) Konzepten,

R. Wilke, Wissenschaft kommuniziert, Wissen, Kommunikation und Gesellschaft, https://doi.org/10.1007/978-3-658-36704-6_1 
Methoden und Ergebnissen, vor allem auf den Verstehensprozess der anderen. ${ }^{1}$ Vornehmlich werde ich in dieser Arbeit daher, am Beispiel des "Group-Talks“ einer von mir beobachteten interdisziplinären Forschungsgruppe, die ,Hürden“ und ,Gräben' sowie die ,Leitern' und ,Brücken' fokussieren, die Prozesse der Kommunikation als Verstehensprozesse in der interdisziplinären Wissenskommunikation charakterisieren.

Mit Kommunikation möchte ich im vorliegenden Kontext zunächst allgemein all jene Vorgänge intersubjektiver Wechselwirkung bezeichnen, die sowohl den verbalen (Sprache), paraverbalen (Sprechweise) und nonverbalen (Mimik und Gestik) Austausch von (subjektivem und objektivem) Sinn, als auch die Chance der gleichzeitigen Erzeugung von (intersubjektivem) Verstehen und (objektiviertem) Wissen umfassen. ${ }^{2}$ Mit dem spezifischeren Begriff der Wissenskommunikation bezeichne ich solche Kommunikationsprozesse, in denen Forscher/-innen ihre z. T. subjektiv gewonnenen wissenschaftlichen Erkenntnisse, vor allem Forschungsansätze, -methoden und/oder -ergebnisse, anderen Wissenschaftler/-innen (in Kopräsenz) vorstellen und mit ihnen diskutieren. Solche Kommunikation ist Gegenstand dieser Arbeit.

Die dieser Dissertationsschrift zugrundeliegende Hauptthese besagt, dass das relativ junge, interdisziplinäre Forschungsfeld der Computational Neuroscience (im Folgenden CNS) praktisch aus einer spezifischen Form kommunikativen Handelns resultiert, durch die es im Interaktionsprozess (re-)produziert wird. Innerhalb dieses kommunikativen Vollzugsprozesses manifestieren sich, gleichsam ko-evolutionär oder dialektisch, sowohl die inkrementelle Ausdifferenzierung und Stabilisierung (Institutionalisierung) einer spezifischen Kommunikationsweise, als auch das korrespondierende wissenschaftliche Feld (Bourdieu und Wacquant 1996) selbst. Beides wird in den Sozialwissenschaften meist diskret

\footnotetext{
${ }^{1}$ Einen ähnlichen Kommunikationsbegriff entwickelt bereits Wilhelm von Humboldt. Er weist darauf hin, dass Sprechen dem intersubjektiven Verstehen diene (Welbers 2001, 574) und dabei vor der Herausforderung stehe, dass ,,aller objectiven Wahrnehmung unvermeidlich Subjectivität beigemischt ist" (v. Humboldt, Grundzüge des allgemeinen Sprachtypus, zitiert nach (Welbers 2001, 576)). Einen ähnlichen Kommunikationsbegriff benutzt auch Margaret Mead in The Small Conference (M. Mead 1968, 5). Allerdings werde ich mich in dieser Arbeit an dem sozialtheoretisch wie methodologisch elaborierten Kommunikationsbegriff des Kommunikativen Konstruktivismus (im Folgenden KoKo; (Knoblauch 2013) (Knoblauch 2017)) orientieren, jedoch ohne diesen voll auszuschöpfen (vgl. Fußnote 2).

${ }^{2}$ Der hier umrissene Kommunikationsbegriff wird im weiteren Verlauf dieser Arbeit noch detaillierter ausbuchstabiert werden (siehe insbesondere Kapitel 2). Er zeigt sich aber bereits hier charakteristisch, nämlich weit und eng zugleich: Einerseits umfasst er mehr als nur Lautsprache (weit wie im KoKo), andererseits exkludiert er für den vorliegenden Zweck weitgehend solche Formen, die Kopräsenz nicht voraussetzen (enger als im KoKo).
} 
betrachtet, wohingegen meine kommunikativ konstruktivistisch informierte Fallanalyse die Frage aufwirft, ob eine dichotome Betrachtung dem tatsächlichen empirischen Phänomen angemessen wäre.

Eine wichtige Rolle für den von mir vor dem Hintergrund dieser These gewählten Forschungsansatz spielt eine Beobachtung, die bereits aus der Soziolinguistik bzw. der kulturanthropologischen Ethnographie der Kommunikation (Hymes 1964) und den hier anschließenden soziologischen Ansätzen bekannt ist, nämlich dass Kommunikation häufig weder spontan noch zufällig verläuft, sondern Muster, Formen und Gattungsmerkmale aufweist, derer sich Sprecher/innen bedienen, um situationsangemessen miteinander kommunizieren zu können. Eine (sprach-)soziologische Ausarbeitung erfuhr diese Idee in den Arbeiten von Thomas Luckmann, der Mitte der 1980er Jahre, aufbauend auf Dell Hymes und anderen, die wissenssoziologische Gattungsanalyse (im Folgenden GA) entwickelte (Luckmann 1986). (Für eine ausführliche Darstellung der GA siehe Kapitel 3.)

In der GA wird davon ausgegangen,

dass kommunikative Gattungen gleichsam die Inseln im Strom kommunikativen Handelns bilden [...] [und] dass in verschiedenen institutionellen Zusammenhängen nicht nur besondere Gattungen vorgezogen werden, sondern dass sie sich durch die Verwendung solcher Gattungen geradezu definieren lassen (Luckmann und Knoblauch 2000, 544).

Dass (soziale) Wirklichkeit, wie ich auch in meiner Haupthese annehme, in Interaktionsprozessen erzeugt und institutionalisiert wird, ist eine aus der Wissenssoziologie bzw. dem Sozialkonstruktivismus (im Folgenden SoKo; (Berger und Luckmann 1986/1969[engl. 1966])) bereits wohlbekannte Erkenntnis. Dass diese Erzeugung maßgeblich durch kommunikatives Handeln prozessiert wird, ist spätestens seitdem (im Gegensatz zu Luckmanns Ansatz nicht länger sprachsondern kommunikationssoziologischen) KoKo (Keller, Knoblauch und Reichertz 2013, Knoblauch 2017) bekannt. Zentral in meiner Analyse ist daher die spezifische Form des kommunikativen Handelns, das besondere Format, in dem die CNS, als relativ junger und interdisziplinärer Forschungskontext von den disziplinär heterogenen Wissenschaftler/-innen im Feld kommunikativ (re-)konstruiert wird. Ich werde argumentieren, dass sich die spezifische Form der Wissenskommunikation hier nicht zufällig als „Group-Talk“ beobachten lässt, sondern dass sich die CNS selbst im Rahmen ihres institutionellen Zusammenhangs durch diese spezifische Kommunikationsform (Lettkemann und Wilke 2016) definieren lässt. 
Group-Talk ist die Feldbezeichnung für die wöchentlichen Arbeitstreffen einer von Kolleg/-innen und mir teilnehmend beobachteten CNS-Forschungsgruppe. Die strukturelle Rekonstruktion des Group-Talks als von mir fokussierter Forschungsgegenstand ist die zentrale Absicht dieser Dissertationsschrift. Analytisch werde ich ihn als kommunikative Gattung betrachten. Die GA erlaubt mir, das Kommunikationsgeschehen im Group-Talk zugleich im Rahmen gesellschaftlicher Strukturkontexte zu besehen, um so die Frage zu beantworten, auf welche Weise und für welche Probleme konkret das Format eine wesentliche Kommunikationslösung darstellt. Gerade hierin besteht der methodologisch-heuristische Wert einer kommunikationssoziologischen GA für die vorliegende Arbeit: Sie ermöglicht es, den reinen Situationalismus, der etwa für die Ethnomethodologie (Garfinkel 1984[1967]) prägend ist, zu überschreiten und die soziologische Mikroanalyse (Handeln), vor dem Hintergrund einer fokussiert-ethnographischen Feldstudie, mit gesellschaftlichen Emergenzphänomenen auf Meso- und Makroebene (Baur, et al. 2016) zu verknüpfen. Erst diese systematische Verbindung des direkt beobachtbaren Wie der Kommunikation mit dem durch ethnographisches Wissen rekonstruierten Warum, überschreitet den Horizont reiner Beschreibung und führt so in die Bereiche des soziologischen Verstehens und Erklärens (Weber 1922, 1).

\subsection{Forschungsfeld und -design}

Die Gelegenheit, entsprechende Kommunikationsvorgänge in einem interdisziplinären Forschungskontext selbst beobachten zu können, ergab sich für mich durch meine Tätigkeit als wissenschaftlicher Mitarbeiter in dem für dreieinhalb Jahre (2013-2017) durch die Deutsche Forschungsgemeinschaft (DFG) geförderten Forschungsprojekt: Bildkommunikation in der Wissenschaft am Fallbeispiel der Computational Neuroscience (Leitung: Hubert Knoblauch) ${ }^{3,4}$. Diese Position konnte ich nach Abschluss meines Masterstudiums in Soziologie mit der Abgabe

\footnotetext{
${ }^{3}$ Weiterführende Informationen siehe hier: http://gepris.dfg.de/gepris/projekt/230927066.

${ }^{4}$ An dieser Stelle möchte ich mich sehr herzlich bei meinem Kollegen Eric Lettkemann für die ausgezeichnete und lehrreiche Zusammenarbeit im Projekt bedanken. Mein Dank gilt außerdem den studentischen Mitarbeiter/-innen im Projekt (Jakob Gerber, Anja Schünzel, Julia Rothenburg und Arne Janz) sowie last but not least unserem Projektleiter Hubert Knoblauch.
} 
meiner Master-Thesis zum Thema Bilder der Wissenschaft. Die kommunikative Konstruktion von (wissenschaftlicher) Wirklichkeit (Wilke 2013) einnehmen. ${ }^{5}$

Das Projekt Bildkommunikation ist im Bereich der wissenssoziologischen Kommunikationsforschung anzusiedeln. Ursprünglich entstand der DFGForschungsantrag im Nachgang einer am Fachgebiet durchgeführten Diplomarbeit, die sich bereits empirisch mit der CNS beschäftigt hatte. Aufbauend auf diesen Vorarbeiten von Lisa-Marian Schmidt (Schmidt 2013), hatte Hubert Knoblauch anschließend den DFG-Antrag zur Bildkommunikation entwickelt. ${ }^{6}$ In dem Projekt übernahm ich, gemeinsam mit Eric Lettkemann, die Fokussierte Ethnographie (Knoblauch 2001). Dabei stellte sich heraus, welche hervorgehobene Rolle der Group-Talk als wöchentliches Forschungsgruppentreffen für die beobachtete Gruppe und die Produktion ihres spezifischen Wissens im Feld der CNS spielte.

\subsubsection{Wissenschaft und Wissenskommunikation}

Die im Rahmen der Bildkommunikation fokussierte CNS ist ein gutes Beispiel für den Übergang der Wissenschaft zu dem, was in wissenschaftssoziologischen Zeitdiagnosen als ,Modus 2' (Gibbons, et al. 1994) bezeichnet wird. Diese

\footnotetext{
${ }^{5}$ Zuvor war ich bereits während meines Studiums für ca. drei Jahre als Tutor am Fachgebiet von Hubert Knoblauch, Allgemeine Soziologie, insbesondere Theorie moderner Gesellschaften, an der Technischen Universität Berlin beschäftigt. In dieser Zeit war ich zum einen damit betraut, die Lehre im Rahmen des Tutoriums für Erst- und Zweitsemesterstudierende zu organisieren bzw. durchzuführen. Zum anderen erlaubte mir diese Tätigkeit auch interessante Einblicke in die Forschung sowie die Methoden- und Theorieentwicklung am Fachgebiet. Insbesondere mit Videographie, Wissenssoziologie und dem KoKo durfte ich mich dabei intensiver auseinandersetzen. Maßgeblich hierdurch wurden die Weichen für meine Herangehensweise als Novize im Projekt Bildkommunikation - und darüber hinaus, für meine Forschungsperspektive bis heute - gestellt.

6 „Ursprüngliches Ziel des Projekts war es, die Entstehungsprozesse neurowissenschaftlicher Visualisierungen, u. a. von fMRT-Bildern, zu begleiten und ihnen über die einzelnen Instanzen der Wissenskommunikation von Wissenschaftler/-innen hinweg zu ,folgen “ (Bildtrajekte). Noch als uns ein entsprechender Feldzugang im Bereich der Computational Neuroscience (CNS) erschlossen wurde, stellte die Optimierung entsprechender Bildgebungsverfahren einen wesentlichen Forschungsgegenstand innerhalb der Forschungsgruppe dar, die ausgewählt wurde. Als das Projektteam im Dezember 2013 die Arbeit schließlich aufnahm, hatten sich die Forschungsschwerpunkte der einzelnen Teilprojekte der Forschungsgruppe allerdings stark verändert, sodass auch die ursprünglichen Fragestellungen und Zielsetzungen bzgl. der Bildtrajekte von fMRT-Bildern und ähnlichen Visualisierungen, gemäß den aktuellen Schwerpunkten im Feld, angepasst wurden." (Knoblauch, Wilke und Lettkemann 2017, 2).
} 
Entwicklung ist davon gekennzeichnet, dass die wissenschaftliche Wissensproduktion von klassisch abgrenzbaren Einzeldisziplinen (Modus 1) zunehmend auf interdisziplinäre ${ }^{7}$ Forschungsgruppen (Modus 2) (Gibbons, et al. 1994, 3ff) übergeht, die insbesondere komplexe und gesellschaftlich relevante Problemstellungen fokussieren (Stichweh 1979, 94). Dabei wird die ,alte', in geschlossenen Fachgemeinschaften und auf Grundlage eines sich nach außen abgrenzenden, homogenen Fachvokabulars prozessierte, disziplinäre Wissensproduktion (ebd., S. 83), von einer neuen Produktionsweise des Wissens abgelöst, die durch die Diversifizierung der Ausdrucks- und Auslegungsschemata und eine zunehmende Diskursivierung gekennzeichnet ist. Diese Entwicklung führte, seit den frühsten Anfängen in den 1960er Jahren, wohin auch die Pionierarbeiten der CNS verweisen, zur raschen Zunahme von gänzlich neuen Forschungsfeldern.

Die CNS stellt damit auch ein Beispiel für die gesamtgesellschaftlich gewachsene Bedeutung der Kommunikation dar, die im Rahmen der Коттиnikationsgesellschaft (Knoblauch 2017, 329ff) zentral verhandelt wird. Dieser Bedeutungszuwachs ist seinerseits in Differenzierungsprozesse eingebettet, die, vor der Wissenschaft, bereits im Alltag beobachtet werden konnten: Schon seit Beginn des 20. Jahrhunderts, insbesondere aber nach dem Zweiten Weltkrieg, scheint der Alltag durch Desintegration charakterisiert (Simmel 1903[2016], Berger, Berger und Kellner 1975, Schulze 1992[2005]), während die Wissenschaft seit den 1960er Jahren zunehmend durch Innendifferenzierung (Stichweh 1979, 82ff) gekennzeichnet ist. In Alltag und Wissenschaft wuchs seither, im Kontext einer dabei zunehmend als komplex wahrgenommenen, emanzipatorisch thematisierten Umwelt (Beck 1986) und vor dem Hintergrund eines daraus hervorgegangenen forschungspolitischen Interdisziplinaritäts-Imperativs (Metzger und Zare 1999, Mansilla, Irwin und Gardner 2006), die Notwendigkeit von Kommunikation und neuen Kommunikationsweisen (siehe Abschnitt 2.2).

\footnotetext{
${ }^{7}$ Während in der Wissenschaftssoziologie keine einheitlichen Definitionen vorliegen (Schützenmeister 2008, 33, Maasen, Lengwiler und Guggenheim 2006, 394), verstehe ich a) unter Disziplinarität die Produktion von Wissen auf Grundlage eines strukturell geschlossenen, wissenschaftlichen Ausdrucks- und Auslegungsschemas, b) unter Interdisziplinarität eine Form der Wissensproduktion, die unterschiedliche wissenschaftliche Ausdrucks- und Auslegungsschemata kombiniert, sowie c) unter Transdisziplinariät eine Form der Wissensproduktion, die auch nicht-wissenschaftliche Ausdrucks- und Auslegungsschemata integriert. Ausschlaggebend für meine Definitionen ist also der Kode der jeweiligen Kommunikation bzw. die grundlegende Annahme, dass Wissen in (unterschiedlichen) Kommunikation(sweisen) produziert wird.
} 


\subsubsection{Computational, interdisziplinär, kommunikativ}

In der Wissenschaft verdient die Entwicklung des Personal Computers (PC) dabei besondere Beachtung, die ihrerseits zum einen die Wissensproduktion revolutionierte und andererseits dazu beitrug, dass Wissenschaft bzw. wissenschaftliches Wissen heute vielfach (inter- und transdisziplinär) kommuniziert wird. Computertechnologische Infrastrukturen und komplexe Informatik erweisen sich seit den 1950er Jahren von rasant wachsender Bedeutung für das Wissenschaftssystem, sowohl als innenstrukturelles, subdisziplinäres Differenzierungsmerkmal (z. B. zwischen Hirnforschung und CNS) als auch als (re-)integrativer Konnex. Dieser Doppelcharakter lässt sich zum einen wissenschaftssoziologisch anhand eines Differenzierungsmodells erklären: Dadurch, dass sich Subdisziplinen, wie sie sich z. B. in den seither vielerorts gegründeten Computational Departments klassischer Disziplinen manifestieren, entlang der Außengrenzen ihrer (Herkunfts-) Disziplinen bzw. an den Rändern vom deren institutionalisiertem Wissens- und Umweltausschnitt (Stichweh 1979, 85 f.) bilden, gehen sie häufig mit personeller und thematischer Interdisziplinarisierung einher (ebd., S. 94 f.). Daher führt diese Form der desintegrierenden Innendifferenzierung letztlich selbst wieder zur Reintegration der disziplinären Spezialdiskurse, in die die Wissenschaft im Prozess ihrer Primärdifferenzierung in Einzeldisziplinen zerfällt (ebd., S. 95). Dies gilt auch und insbesondere für Computational Sciences (Gramelsberger 2015), die, wie z. B. die CNS, zahlreiche Disziplinen überspannen und diese dabei, im Sinn einer gemeinsamen globalen Problemstellung, reintegrieren.

Dieser, im Sinne von Innendifferenzierung und Reintegration (und folglich: Diskursivierung), nacheinander in entgegengesetzte Richtungen wirkende Doppelcharakter der Einführung des Computers und insbesondere des PCs in die Wissenschaft lässt sich andererseits auch empirisch nachzeichnen, anhand der gewachsenen Bedeutung dieser Technologie in der wissenschaftlichen Praxis. Der PC und die fortschreitende Entwicklung von Hard- und Software haben nicht nur dazu beigetragen, analoge Praktiken in digitale zu verwandeln, wie in der Produktion (Slautterback und Werther 1984), der Buchhaltung (Cerullo 1980) oder dem Schreiben und Kommunizieren (Warschauer 2007). Vielmehr machen moderne PCs multi-variable und interdisziplinäre Forschungen möglich, die zuvor unmöglich waren. Dies betrifft vor allem den Bereich der numerischen Modellierung von globalen Systemen, der auf PCs basiert und heute aus zahlreichen Subdisziplinen innerhalb klassischer Disziplinen nicht mehr wegzudenken ist (Gramelsberger 2010, 2015): Hier bilden Computer und entsprechende Expertise buchstäblich den epistemischen Kern dieser ,neuen' Computational Sciences; etwa in der 
Geophysik (Computational Geophysics), der Astrophysik (Computational Astrophysics), der Linguistik (Computational Linguistics), der Wirtschaftswissenschaft (Computational Economics) usw.

Es ist deutlich, dass es durch Computer und informatische Expertise auf vielen Wissenschaftsfeldern zum Wandel bzw. zur völligen Neuausrichtung klassischer Disziplinen (Malina 2010) sowie zur Ausbildung neuer Forschungsfelder und (Sub-)Disziplinen gekommen ist (Innendifferenzierung). Diese Entwicklung zeichnet Gabriele Gramelsberger (2010) exemplarisch anhand des Wandels der beobachtungsbasierten Wettervorhersage zur numerischen, computerbasierten Meteorologie nach. Dabei beschreibt sie allerdings auch ,die enorme Vereinheitlichung der Forschungspraxis" (ebd., S. 143), die mit dem Einzug des Computers in die Wissenschaft allgemein einhergeht (Reintegration). Vor seiner Einführung konnte man, ,an den Tätigkeiten der Forscher meist ablesen, welcher Disziplin sie angehörten“, heute allerdings, so die Wissenschaftsphilosophin weiter, „lässt sich dies für die Computational Sciences nicht mehr erkennen. Ob ein Biologe, ein Klimaforscher oder ein Physiker vor dem Computer sitzen und ihrer Arbeit nachgeht [sic!; R.W.], lässt sich von außen betrachtet nicht entscheiden. Alle tun dasselbe, sie schreiben Codes." (ebd.). Vor diesem Hintergrund spricht Gramelsberger von einer allgemeinen Transformation der Wissenschaft (Gramelsberger 2015).

Dabei okkupieren Computerwissenschaftler/-innen heute innerhalb der gewandelten (Sub-)Disziplinen (Stichweh 1979, 84ff) selbst eine relevante Unsicherheitszone (Crozier und Friedberg 1979, 49ff). Sie leisten dort personell die Integration, wo die Übersetzung komplexer multi-disziplinärer Forschungsgegenstände in Computercode durchaus noch genuin computerwissenschaftlicher Expertise bedarf. Die Globalität entsprechender Modelle lässt sich am Beispiel der CNS verdeutlichen: Sie erstrecken sich von Ionen über Transmitter und Rezeptoren, Neuronen, Netzwerke und Hirnregionen bzw. neuronale Systeme, bis hin zu Verhalten und Denken. Dabei verknüpfen sie zahlreiche Disziplinen wie Physik, Chemie, Biologie, Computerwissenschaft, Neurologie und Psychologie (Lytton 2002, 16). In der beobachteten CNS-Forschungsgruppe verschwammen die Grenzen zwischen den einzelnen beteiligten Disziplinen allerdings nicht nur in der Außenbetrachtung (Lynch 1991, 53), worauf sich Gramelsberger oben bezieht, sondern, wie sich zeigen wird, auch in der Forschungsbiographie und -persönlichkeit der einzelnen Wissenschaftler/-innen selbst, die sich z. $B$. von der Psychologie oder der Physik über die Computerwissenschaft oder umgekehrt zur CNS weiterentwickelt hatten. In diesem Sinn stellt nicht nur die CNS eine interdisziplinäre oder konkreter: eine durch den Computer verknüpfte synthetische Wissenschaft (Knoblauch und Wilke 2020) dar, sondern auch die Computational 
Neuroscientists selbst, die wir beobachten konnten, waren an dem Ideal orientiert, die verschiedenen Spezialwissensbestände der einzelnen an der CNS beteiligten Disziplinen in ihrer eigenen Forschungspersönlichkeit gleichsam zu vereinen.

Das neue Wissen, das in diesem Kontext erzeugt wird, stellt dabei nicht nur substanziell, sondern auch prozessual, d. h. kommunikativ, hohe Ansprüche an die betroffenen Wissenschaftler/-innen: Es muss, im Sinne der Reintegration, permanent übersetzt werden (Diskursivierung). Daher wächst, zugleich mit der Computerisierung und der Interdisziplinarisierung der wissenschaftlichen Wissensproduktion, die Notwendigkeit der Verständigung über eine wachsende Zahl beteiligter Disziplinen hinweg: Wer immer spezifischeres Wissen in zunehmend differenzierten Subsphären produziert und dieses daraufhin an breitere Communities rückkoppeln muss oder möchte, der hat auch eine immer schwierigere Kommunikationsaufgabe zu bewältigen. Für die wissenschaftliche Praxis, für Institutionen und individuelle Akteure, bedeutet das unweigerlich neue Herausforderungen durch zusätzliche Kommunikationsprobleme (Balck 2016): Im Kontext von Computer-getriebener Innendifferenzierung bzw. Subdisziplinierung und einem forschungspolitischen Interdisziplinaritäts-Imperativ, der praktisch aus gesamtgesellschaftlichen Enttraditionalisierungs- und Emanzipationsprozessen sowie der Risikogesellschaft (Beck 1986) hervorgegangen ist, muss die Bedeutung des eigenen, fach- bzw. feldidentitären Wissens, das in Disziplinenüberschreitenden Diskursen mehr oder minder, in der Fremde " verbleibt, daher immer wieder neu erklärt, ausgehandelt und legitimiert werden. So entstehen neben den neuen interdisziplinären Feldern schließlich aber auch neue Problemgetriebene Kommunikationslösungen, wie der Group-Talk, um Wissen, das im ,Modus 2‘ (Gibbons, et al. 1994) produziert wurde, auch kommunizieren zu können.

Die CNS stellt auch hierfür ein besonders gutes Beispiel dar. Entsprechend der Interdisziplinarität des Felds zeigte sich in der vorliegenden Untersuchung, sowohl intern als auch in der Außenkommunikation, ein sehr hoher Kommunikationsbedarf, der vor allem in dem hier zentral thematisierten Group-Talk der Forschungsgruppe zum Ausdruck kam: Hier versuchten die Wissenschaftler/-innen der verschiedenen Disziplinen der Gruppe, ausgehend von ihren heterogenen Grundannahmen, auf Grundlage von Computermodellierungen, die Erforschung neuronaler Funktionen auf allen oben genannten Ebenen ihres Forschungsgegenstands voranzutreiben. ${ }^{8}$ Zugleich sollten die so im gemeinsamen Schnittfeld

\footnotetext{
${ }^{8}$ Die Erforschung der fundamentalen Prinzipien des Seins wird hierbei zu einer Computerwissenschaft. Eine sozialwissenschaftliche Kritik daran findet sich u. a. in (Choudhury und Slaby 2012).
} 
erlangten Erkenntnisse auch in der Künstliche Intelligenz-Forschung (im Folgenden KI-Forschung) angewendet werden können. Kommunikation kam dabei die entscheidende Bedeutung zu, nicht auf Grundlage eines vorgängig geteilten, disziplinären Wissens- und Ausdrucksschemas, sondern ausgehend von formaler Heterogenität gemeinsam, neues Wissen zu erzeugen.

\subsubsection{Das Feld der CNS}

Entsprechend geht bereits die Etablierung des Begriffs Computational Neuroscience maßgeblich auf Gespräche, Publikationen und Konferenzen zurück, die ab 1985 von Eric L. Schwartz, Professor für kognitive und neuronale Systeme, Computerwissenschaft sowie Neurobiologie und -anatomie initiiert wurden, um Gehirntheoretiker/-innen verschiedenster Provinienz mit Computerwissenschaftler/-innen ins Gespräch zu bringen und dabei den Begriff Computational Neuroscience zu definieren sowie das entsprechende Forschungsfeld zu umreißen. Die grundlegende, technisch-infrastrukturelle Voraussetzung für das neue Forschungsfeld stellte dabei, darauf weist schon das englische Adjektiv computational hin, die zeitgleiche Computer-Revolution der 1980er Jahre dar. Zum damaligen Zeitpunkt zogen erstmals flächendeckend PCs in den Wissenschaftsalltag ein (für die Computational Chemistry siehe (Levy 1988)). In einer frühen Selbstbeschreibung der CNS heißt es bezugnehmend auf diese Entwicklung:

[T]echnical achievements in designing fast, powerful, and relatively inexpensive computing machines have made it possible to undertake simulation and modeling projects that were hitherto only pipe dreams (Churchland, Koch und Sejnowski 1990, 47).

So ermöglichen moderne PCs seither eine relativ ressourcenschonende Modellierung komplexer neuronaler Prozesse, wie sie für die Performanz des (menschlichen) Hirns, im Rahmen moderner Neurotheorien im Feld der CNS, angenommen werden. Zuvor, etwa seit den 1940er Jahren, waren für komplizierte Berechnungen noch gigantische Anlagen wie der Großrechner „Mark 1“ notwendig, die viel Platz benötigten und aus Kostengründen nur wenigen Einrichtungen weltweit zur Verfügung standen. Vor 1945 war an die Entwicklung und Verarbeitung heutiger neurowissenschaftlicher Modelle überhaupt noch nicht zu denken. Erst seit den 1980er Jahren konnten entsprechende Softwareprogramme zunehmend auf relativ günstigen PCs abgespielt werden, die dann bald zur landläufigen Büro- bzw. Laborgrundausstattung in der Wissenschaft zählten (Levy 
1988, 167). Erst dadurch konnte in der Folgezeit ein breiter interdisziplinärer Diskurs angeregt werden, aus dem die gegenwärtige CNS, als moderne, Disziplinen-überspannende, computergestützte Hirnforschung resultierte.

In einem Sammelband, der aus einer von Schwartz organisierten Konferenz im kalifornischen Carmel hervorgegangen ist, schreibt der Neurobiologe Donald Perkel entsprechend: "“'Computational neuroscience” in early 1987 is very much a term in search of a definition, a field begging for a map and boundaries" (1990, 38). Als wir, über 25 Jahre später, mit unserer Forschung im Projekt Bildkommunikation begannen, hatte die CNS zwar längst begonnen, sich über die Grenzen der USA hinaus zu etablieren, dennoch ließ sich noch immer beobachten, dass sie sich stets in einem kommunikativen Aushandlungsund Institutionalisierungsprozess befand und ihre akademische Verankerung als eigenständige Disziplin, gemessen an CNS-Studiengängen in Deutschland (Stand 2020: zwei Masterstudiengänge), noch nicht weit vorangeschritten war. Vielmehr erwies sich die, aus der ursprünglichen Grundkonstellation einer kommunikativ prozessierten Orts- und Wesensbestimmung sowie vor dem Hintergrund der Multidisziplinarität des CNS-Ansatzes hervorgegangene, heterogene Wissensordnung, die im Feld noch immer zahlreiche Fachdisziplinen überspannt, nicht nur für die soziologischen Ethnographen, sondern, wie ich zeigen werde, auch für die genuinen Feldteilnehmer/-innen, als überaus herausfordernd.

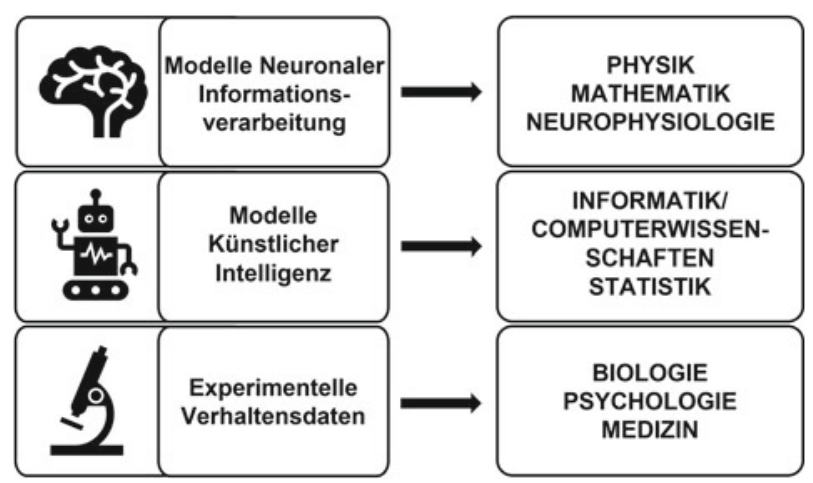

Abbildung 1.1 Das Feld der CNS mit den typischen Schwerpunkten: Theoretische Hirnforschung, Künstliche Intelligenz Forschung und experimentelle Verhaltensforschung 
Die CNS verfolgt heute, gemäß unseren eigenen Beobachtungen, drei genuine Aufgabengebiete (Abbildung 1.1): Erstens möchte sie die neurobiologischen Grundlagen des Gehirns mittels mathematischer Computermodelle besser verstehen lernen. Hierbei geht es darum, zum Feld der Hirnforschung entscheidende Erkenntnisfortschritte beizutragen. Zweitens sind Teilbereiche der CNS der Aufgabe gewidmet, die Performanz des Gehirns in Maschinen nachzubauen. Sie stellt somit sowohl einen computerwissenschaftlich basierten Ansatz zur Hirnforschung als auch einen (neurobiologisch inspirierten) Zweig der KI-Forschung dar. Im Rahmen der beobachteten Forschungsgruppe sind Wissenschaftler/-innen im Feld der CNS drittens mit Methoden der (Re-)Analyse verhaltensexperimenteller Daten beschäftigt (vgl. Perkel (1990)). Die CNS fußt dabei, als maßgebendes Erkenntnismittel (Bechtel in press), als wesentliche Referenz der Kommunikationsarbeit (Knoblauch 1996) und als kommunikative Ressource (Bergmann 1988a), auf computergestützte numerische Modelle.

\subsubsection{Die Kommunikation von Wissen in der CNS}

Neben die epistemischen Zielsetzungen tritt, vor diesem Hintergrund der CNS, die kommunikative Aufgabe der interdisziplinären Objektivierung, Vermittlung und CNS-spezifischen Institutionalisierung des in individuellen Forschungsprojekten erlangten, neuen (subjektiven) Wissens. Die Verkehrssprache der CNS ist dabei Englisch, eine deutschsprachige CNS-Forschung gibt es nicht, da sämtliche einschlägige Publikationsorgane international sind. Auch der GroupTalk der beobachteten Forschungsgruppe wurde aufgrund ihrer internationalen Zusammensetzung auf Englisch abgehalten. ${ }^{9}$ Das Repertoire der Kontaktsprache (Abschnitt 4.2) umfasste dabei, neben sehr allgemeinen Sprechweisen, auch eine Vielzahl von Fachtermini der verschiedenen Herkunftsdisziplinen, die durchaus nicht allen Teilnehmer/-innen geläufig bzw. verständlich waren. Im Feld zeigte sich in diesem Kontext, dass nicht allein die Erkenntnis, sondern auch deren Kommunikation in der CNS maßgeblich auf PCs basierte. Eine besondere Rolle spielte der Rechner dabei als Kommunikationsmedium, das es erlaubt, Daten und Forschungsergebnisse zu visualisieren (Amann und Knorr Cetina 1988).

\footnotetext{
${ }^{9}$ Der Forschungsgruppenleiter teilte uns mit, dass kurz vor unserer Forschung die Umstellung von Deutsch auf Englisch im Group-Talk erfolgte, um der gewachsenen Internationalisierung der Gruppe Rechnung zu tragen.
} 


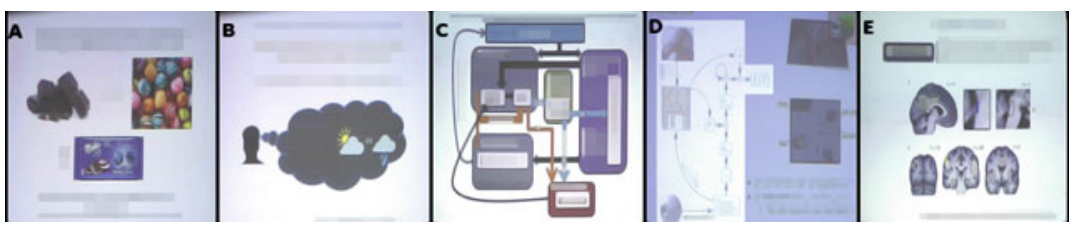

Abbildung 1.2 Typische Visualisierungen auf Digitalfolien (anonymisiert) im Group-Talk: Alltagsbilder $(\mathrm{A}+\mathrm{B})$, kybernetische Darstellungen $(\mathrm{C}+\mathrm{D})$, statistische Visualisierungen (E)

Visualisierungen von Forschungsansätzen, konkreten Modellen und Ergebnissen dienen in der CNS als wesentlicher kommunikativer Bezugspunkt der Fachvertreter/-innen der unterschiedlichen Domänen. Wie sich in unserer Forschung gezeigt hat, ist die Verwendung verschiedener visueller Kompositionen (Abbildung 1.2), im Sinne einer Bild-Kommunikation (Abschnitt 4.2), eines der bedeutendsten und gebräuchlichsten Verständigungsmittel innerhalb der CNS. Digitale bzw. digitalisierte Visualisierungen werden hier zur Versinnbildlichung von Forschungsfragen ebenso häufig herangezogen, wie zur Illustration der Nützlichkeit neuer Auswertungsverfahren oder, wie im Bereich der Simulation, zur Evidenzerzeugung für konkrete Forschungsergebnisse. Darüber hinaus dienen sie vorgängig bereits als Erkenntnismittel und schließlich als Ressource für die Außenkommunikation (Wilke und Hill 2019).

In unserer Beobachtung zeigte sich, dass die entwickelte und angewendete gemeinsame Sprache in der Forschungsgruppe nicht als pidgin (Galison 1994), d. h. als ein in seiner semantischen Ausdrucksvielfalt eingeschränkter Code, der allein speziellen Anlässen dient, verstanden werden kann. Vielmehr erwies sie sich als Lingua Franca, d. h. als linguistisch wie funktional vollwertige Kontaktsprache (House 2003, 557). Das gleiche gilt für die feld-typischen Visualisierungen, die nicht als boundary objects (Leigh Star und Griesemer 1989) begriffen werden können, da sie im Group-Talk in eine Bild-Kommunikation eingebettet werden. Sie dienten so vielmehr dem wechselseitigen Verstehen und repräsentierten für die unterschiedlichen Vertreter/-innen der an der Gruppe beteiligten Disziplinen, anders als ,Grenzobjekte', nicht systematisch unterschiedliche, aus der jeweils subjektiven Perspektive abgeleitete Aspekte, sondern wurden dazu geschaffen, ein gemeinsames Verständnis zu erzeugen.

Im Kontext dieser buchstäblichen Bedeutung von „Group-Talk“, als Verstehens-basiertes Reden-in-Interaktion, beobachteten wir aber auch, dass 
ihre Kommunikationssituation die Forscher/-innen vor große Herausforderungen stellte. Verstehen und Verstanden-Werden verlangten im Group-Talk, neben Bild-Kommunikation und verbaler Kontaktsprache, vor allem ein spezifisches, prozessuales Wissen, das wir präsentational nennen (Wilke, Lettkemann und Knoblauch 2018). Das präsentationale Wissen stellt ,Rezepte' für Performanz (Knoblauch 2007) und Orchestrierung (Schnettler 2007) der verbalen und digitalvisuellen Basiselemente bereit. Es zielt dabei ,nicht auf die Abbildung eines epistemischen Objekts“, sondern erlaubt „Wissen auf eine Weise auf[zuzeigen; R.W.], die es für andere verwendbar macht" (Wilke, Lettkemann und Knoblauch 2018, 246). Das präsentationale Wissen der CNS ist daher elementar für deren Wissenskommunikation, die sich kategorisch von oberflächlichen Kommunikationsweisen unterscheidet. Es dient dazu, den eingesetzten Objektvierungen und digitalen Objektivationen eine Form zu verleihen, die erkannt, verstanden und reproduziert werden kann.

Die Feldbezeichnung „Group-Talk“ ist daher zudem buchstäblich zutreffend, weil hier, neben der gemeinsamen, in Kommunikation prozessierten Etablierung von substantiellem (CNS-spezifischem) und prozessualem (präsentationalem) Wissen, auch die Wissenden selbst in der sowie durch die Gruppenkommunikation sozialisiert wurden. Viele Wissenschaftler/-innen der Forschungsgruppe hatten sich durch ihre Teilnahme an der Gruppe und dem Group-Talk aus ihrer ursprünglichen Disziplin herausentwickelt, wie z. B. eine Bio-Physiker/-in, die in kognitiver Neurowissenschaft promoviert hatte und sich nun als ,Computational Cognitive Neuroscientist" bezeichnete (Exp_11, Z. 38ff), oder ein Informatiker, der seine Ausbildung als ,nicht-linear“ bezeichnete (Exp_02, Z. 216ff) und nun an KI forschte.

\subsubsection{Fokussierte Ethnographie}

Die für die vorliegende Arbeit maßgebliche Datenerhebung im Projekt Bildkommunikation fand im Rahmen der angesprochenen CNS-Forschungsgruppe bzw. während des Group-Talks statt. Die beobachtete Gruppe setzte sich im Erhebungszeitraum aus 20 internationalen Wissenschaftler/-innen (zwei Professoren und 18 (Post-)Doktorand/-innen) zusammen; unter ihnen drei Physiker/-innen, zwei Mathematiker/-innen, fünf Informatiker/-innen, zwei Neurophysiolog/-innen und sieben (Neuro-)Biolog/-innen sowie ein Psychologe. Ca. ein Drittel der Gruppe waren Frauen. In zwei Fällen wurde die Forschungsgruppe von CNSStudierenden besucht, deren Mentor/-in Teil der Forschungsgruppe war. 
Jenseits dieser Zusammenkünfte zeigte sich die Arbeit der Gruppe maßgeblich vom individuellen Forschungsprozess bestimmt. Im Rahmen dieses Forschungsalltags arbeiteten die Forscher/-innen zumeist solitär. Ein Großteil ihrer Forschungsarbeit vollzog sich im Digitallabor (Gramelsberger 2010): in der Interaktion mit ihren Computern, Datenkorpora und Softwareprogrammen. Gerade aber deshalb kam der regelmäßigen Gelegenheit des Austauschs im Group-Talk, wie sich zeigte, eine besondere Bedeutung zu. Er stellte den zentralen Kreuzungspunkt dar, an dem die Vertreter/-innen der verschiedenen Wissenskulturen (Knorr Cetina 2002) sich begegneten und die Externalisierung ihres Forschungsprozesses, die Objektivierung ihrer Erkenntnis, der intersubjektiven Validierung anheimstellten.

Aufgrund dieser Schlüsselrolle stellt der Group-Talk die fokale Situation des fokussiert ethnographischen Forschungsdesigns der vorliegenden Arbeit dar. Wir haben in der Erhebungsphase über den Zeitraum von ca. 14 Monaten an den Treffen der Forschungsgruppe teilgenommen und dabei im Rahmen von 45 (37 Einzelvorträge habe ich selbst beobachtet) teilnehmend beobachteten Group-Talks ca. 14 Stunden audio-visuelle Daten erhoben. Zur Rekonstruktion der Wissensund Erfahrungsstruktur (Knoblauch 2001, 135) der Feldteilnehmer/-innen haben wir zusätzlich 10 Expert/-inneninterviews und 2 Elizitationsinterviews (Knoblauch 2004a, Schubert 2008) von durchschnittlich ca. 90 Minuten Dauer geführt, transkribiert und mittels qualitativer Inhaltsanalyse (Mayring 2010) ausgewertet. Zur Kontrastierung haben wir zudem ca. 9 Stunden audio-visuelle Daten im Rahmen eines interdisziplinären geisteswissenschaftlichen Kolloquiums erhoben. Darüber hinaus war auch ein Dokumentenstudium notwendig, um die spezifischen Inhalte, die im Feld diskutiert wurden, kontextualisieren zu können. $\mathrm{Zu}$ diesem Zweck haben wir uns vor allem mit Selbstthematisierungen der CNS (einen Überblick bieten etwa (Schwartz 1990, Bower 2003, Lytton 2002) sowie mit Publikationen der Feldteilnehmer/-innen beschäftigt.

Unser Forschungsdesign bezeichne ich als fokussiert ethnographisch: Fokussierte Ethnographie (Knoblauch 2001) beschreibt eine Forschungspraxis, die nicht allein in der Soziologie, sondern in zahlreichen sozialwissenschaftlichen Disziplinen ihre Anwendung findet. Maßgeblicher Orientierungspunkt der Methode ist die ethnologische Ethnographie, die vor allem mit dem Kulturanthropologen Bronislaw Malinowski $(1922,1926)$ und seinem Begriff der Teilnehmenden Beobachtung (participant-observation) verknüpft ist. Diese Methode, die es sich zum Ziel setzt, Praktiken und Wissensbestände beforschter Ethnien durch die Teilnahme an deren Alltag zu erkunden, war erfolgreich angetreten, um ,einen Bruch mit einer stubengelehrten „Armchair-Anthropology““" (Amann und Hirschauer 1997, 10) herbeizuführen. In der Folge fand die Ethnographie auch im Rahmen 
der eigenen Gesellschaften, in anthropologischen (Urban Studies), linguistischen (Soziolinguistik) und soziologischen Forschungen (Chicagoer Schule), breite Anwendung, wo Ethnograph/-innen, Sprach- und Sozialwissenschaftler/-innen sich auf den Weg machten, sich in das erweiterte soziale Umfeld zu begeben, um ethnographische Feldforschung buchstäblich ,vor der eigenen Tür ${ }^{\bullet}$ zu betreiben. Diese Bewegung erweist sich, nicht zuletzt im Kontext der für die vorliegende Arbeit zentralen Zeitdiagnose, von besonderer Relevanz, worauf auch Stefan Hirschauer und Klaus Amann hinweisen: „[V]ielschichtig differenzierte Wissensgesellschaften“ (ebd., S. 12), wie westliche Gegenwartsgesellschaften, sind durch eine unüberschaubare Variabilität sozialer Felder und Gruppen charakterisiert, sodass sich die für die Ethnographie ebenso kennzeichnende wie motivierende Befremdung auch innerhalb der eigenen Gesellschaft einstellt. Dies gilt insbesondere für Wissenschaftsethnographien, die von Wissenschaftler/-innen über Wissenschaftler/-innen erstellt werden (Knorr Cetina 2002, Latour 1987, Lynch 1993). Die Pointe ist, dass die Nähe trügt und, wie uns die Science and Technology Studies (STS) lehren (Jasanoff, et al. 1995), ein Blick in die Praxis von Wissenschaft lohnt, um festzustellen, dass deren feldspezifische Besonderheit nicht durch beschreibende Auslegungsschemata, reine (Ethno-)Theorie, zu erfassen ist. Der Schritt aus dem Lehnsessel ist notwendig. Sei es auch nur eine kurze Strecke, die zur ,fremden Sinnprovinz' zu überwinden ist. In diesem Sinn bezeichne ich unsere Ethnographie erstens deshalb als fokussiert, weil ich mich im Projekt, als soziologischer Ethnograph, in der eigenen und somit grundsätzlich vertrauten Gesellschaft bewegt habe. Das bedeutet nicht, wie ich bereits erläuterte, dass soziologischen Ethnograph/-innen ihr Forschungsgegenstand a priori bekannt sei. Im Gegenteil, in der vielschichtig differenzierten Wissensgesellschaft sind ,Welten' häufig buchstäblich nur durch eine einzelne Wand voneinander getrennt. So kann es ähnlich befremdlich sein, innerhalb derselben Universität das Stockwerk zu wechseln, als würde man sich als Anthropolog/-in auf die Reise zu einer weit entfernten Kultur begeben.

Das Attribut „fokussiert“ weist zweitens auf einen weiteren entscheidenden Unterschied zur ethnologischen Ethnographie Malinowskis hin, die sich (ursprünglich) auf (völlig) unbekanntes Terrain begibt. Fokussiert ist die vorliegende Ethnographie auch hinsichtlich der ethnographischen Konzentration auf einen kleineren ,weißen Flecken“ inmitten der ,Landkarte“ einer grundsätzlich bekannten und persönlich sehr vertrauten Umwelt, nämlich der akademischen Wissenschaft und Forschung. Um bei dem obigen Beispiel zu bleiben: Zwar werden sowohl für mich, der ich ,zu Hause ' bleibe, als auch für eine Anthropolog/-in, die sich zu einer unbekannten Kultur aufmacht, Unterschiede und Gemeinsamkeiten zu verzeichnen sein. Allerdings sind diese in beiden Fällen wahrscheinlich 
sehr unterschiedlich verteilt. Während die kulturellen Gemeinsamkeiten gegenüber einer erstkontaktierten Kultur in der Ferne zunächst gering erscheinen mögen, drängen sich diese, durch die sozial-räumliche Nähe impliziert, in der eigenen Gesellschaft auf. Im Zentrum der vorliegenden Ethnographie standen daher, eingedenk des Postulats, sich von scheinbarer Vertrautheit nicht blenden zu lassen, nicht die Universität, die Disziplin, die Interdisziplinarität oder gar die Wissenschaft in toto, sondern konkret die Kommunikationsprozesse im Rahmen der fokalen Situation in der beobachteten CNS-Gruppe. ${ }^{10}$

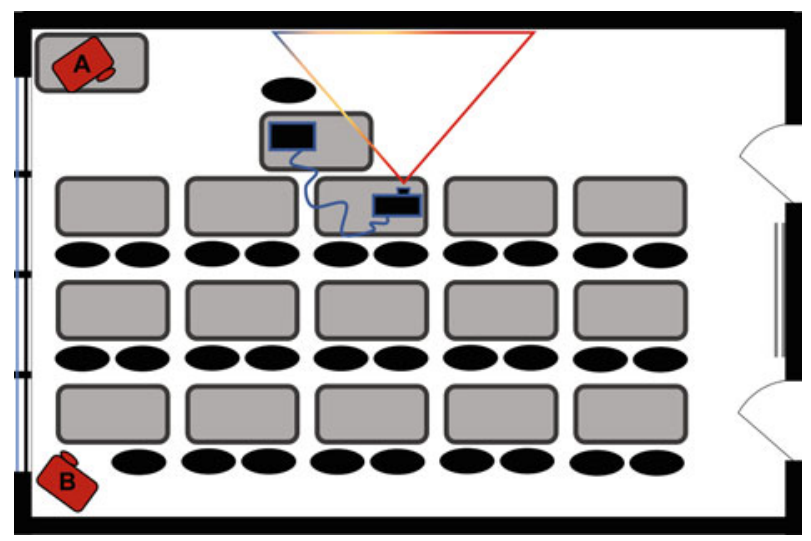

Abbildung 1.3 Schematische Darstellung des räumlichen Settings und der Kamerapositionen: Publikumsperspektive (A) und Sprecherperspektive (B)

\subsubsection{Videographie}

Charakteristisch für die Fokussierte Ethnographie ist drittens die audio-visuelle Methode. Videographie (Tuma, Schnettler und Knoblauch 2013), als Teil der

\footnotetext{
${ }^{10}$ Die folgenden dieses Kapitels, die das Forschungsdesign im Projekt Bildkommunikation zusammenfassen, stellen zum Teil Auszüge einer frühen Version eines Texts dar, den ich später mit Eric Lettkemann (Lettkemann und Wilke 2016) veröffentlicht habe. Für einen Handbuchartikel hatte ich die empirischen Analysen sowie die Beschreibung der Datenerhebungsund Auswertungsverfahren aus meinem Promotionsprojekt beigesteuert. Bei Eric Lettkemann und den Herausgeber/-innen des Sammelbands (Baur et al. 2016) bedanke ich mich für die zahlreichen Anmerkungen zu dem ursprünglichen Textfragment, die in die publizierte Fassung in großem Maße eingeflossen sind. An dieser Stelle bediene ich mich allerdings an Auszügen des ursprünglichen Texts, für den ich allein verantwortlich zeichne, sodass ggf. auch alle Fehler oder Ungenauigkeiten mir zuzuschreiben sind.
} 
Fokussierten Ethnographie, nimmt in ihr einen großen Teil der Datenerhebung und -auswertung ein. Im Projekt Bildkommunikation verwendeten wir, zum Zweck der Videoaufzeichnung des Group-Talks, zwei Digitalkamerasysteme: Erstens einen HD-Camcorder mit Aufsatzmikrophon (Abbildung 1.3, Kameraposition A), den wir auf ein Stativ montierten, sowie zweitens eine Full-HD Action Cam (Abbildung 1.3, Kameraposition B). Letztere eignet sich, aufgrund ihres großen Blickfelds und ihrer Kompaktheit, besonders für die Aufzeichnung aus Sprecher/-innenperspektive. Ihr kleines Gehäuse ermöglichte es, die Reaktivität (Behnke, Baur und Behnke 2006, 249ff) des aufgezeichneten Publikums zu verringern. Den Camcorder mit Stativ platzierten wir im, vom Publikum aus betrachtet, rückwärtigen Teil des Raums, sodass die Aufzeichnung den Raum, die in ihm stattfindende Kommunikation und die dabei zum Einsatz gebrachten technischen Medien (z. B. Laptop, Digitalprojektor) aus Publikumsperspektive erfasste.

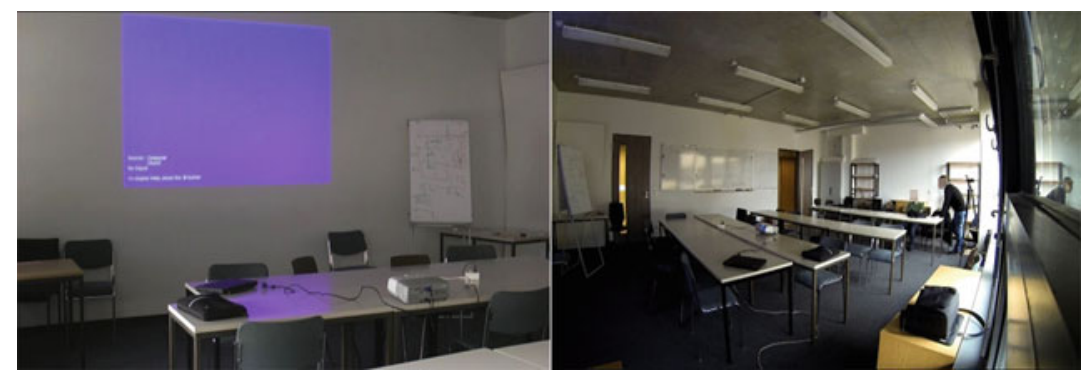

Abbildung 1.4 Räumliches Setting des Group-Talks aus Perspektive der beiden Kamerapositionen (links Perspektive B, recht Perspektive A (vgl. vorhergehende Abbildung)

Räumlich wurde der Group-Talk an einem deutschen Universitätsinstitut, an dem die Forschungsgruppe beheimatet ist, in einem kleinen Seminarraum veranstaltet (Abbildung 1.4; eine ausführliche Darstellung folgt in Abschnitt 4.1.1). Die Sitzordnung entsprach weitgehend einer klassischen Frontalkonstellation, wobei der bzw. die Hauptsprecher/-in i. d. R. vor den anderen Teilnehmer/-innen stand (siehe: Abbildung 1.5, Position 1). Das Publikum saß ihr bzw. ihm in drei Reihen klassischer Seminartische gegenüber (Abbildung 1.5, Position 2). Typischerweise bedienten sich die Hauptsprecher/-innen einer Präsentationssoftware, um, mittels des stationären Digitalprojektors und eines mitgebrachten Laptops (Abbildung 1.5, Position 3), vorentworfene Foliensätze an die dem Publikum gegenüberliegende Wand zu projizieren. 


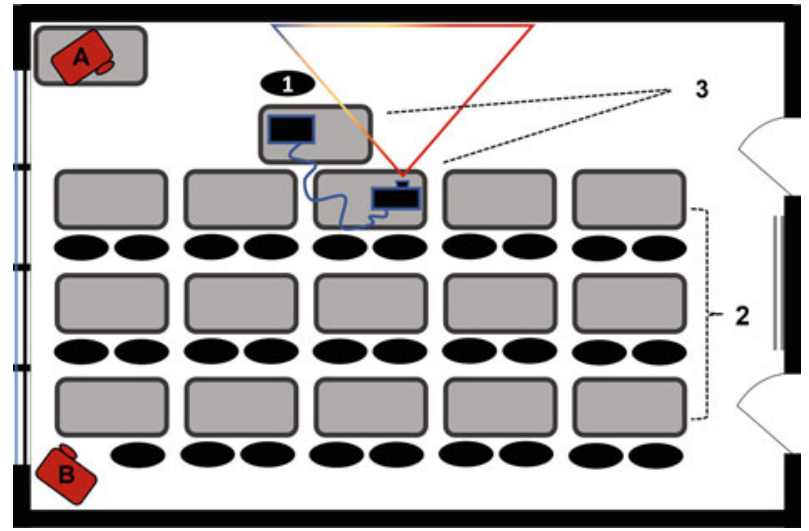

Abbildung 1.5 Eigene schematische Darstellung der Positionen der Hauptsprecher/-in (1), des Publikums (2) und der Technik (3)

In der Aufzeichnung, Aufbereitung und Auswertung der audio-visuellen ,Feldkonserven“ - in der Videographie - kommt ein entscheidendes Merkmal der Fokussierten Ethnographie zum Ausdruck, das sie deutlich von der ,herkömmlichen" Ethnographie unterscheidet: Während man Erstere als besonders „,erfahrungsintensiv“ (Knoblauch 2001, 129) bezeichnen kann, ist Letztere besonders „datenintensiv“ (ebd.). Das bedeutet, anders als in klassisch ethnographisch verfahrenden Studien, kehrten wir, nach relativ kurzen Feldaufenthalten (einige Stunden pro Woche), neben unseren Beobachtungsdaten und Feldnotizen, auch mit einer multimodalen Repräsentation aus dem Feld zurück, die , objektiv “ konservierte, was in der dort fokussierten Situation tatsächlich stattgefunden hat. Die eigentliche Analyse begann für uns daher auch weniger bereits beim Verfassen von Feldnotizen vor Ort, sondern maßgeblich im Rahmen von sich mit den Feldaufenthalten abwechselnden, intensiven Aufbereitungs- und Analyseschritten im Videolabor, in deren Verlauf das erhobene Filmmaterial gesichert, gesichtet und mittels qualitativer Kodierung allmählich für die Analyse , aufgebrochen " wurde. Die Möglichkeit, in dem Material hin und her spulen, es Bild-für-Bild betrachten und die Aufzeichnung an jedem beliebigen Punkt stoppen zu können, ist für die videographische Kommunikationsanalyse (Interaktion und Kommunikation) im Rahmen einer Fokussierten Ethnographie entscheidend. Verbale, non-verbale und paraverbale Kommunikation, d. h. Sprache, Gestik, Mimik und die tonale Modulation der lautsprachlichen Kommunikation sowie - last but not least - der 
Umgang mit Objekten, konnten so gleichermaßen erfasst und in das Zentrum ausgiebiger Feinanalysen gerückt werden (Kapitel 4). Dabei ergab sich häufig erst im Videolabor und bei der Durchsicht des Materials, und nicht etwa bereits vor Ort im Feld, welche Sequenzen sich für unsere Arbeit als besonders vielversprechend erweisen sollten und einer Detailanalyse unterzogen werden mussten. Eine wesentliche Rolle spielte hierbei auch der Einsatz von Videoanalysesoftware. Am Fachgebiet hatte sich zu diesem Zeitpunkt die Software Dartfish (Tuma 2017, 184) etabliert, die ich im Rahmen der Datenaufbereitung intensiv nutzte.

Dartfish stammt aus dem Bereich der Sportwissenschaft und kommt dort in der Bewegungsanalyse zum Einsatz (ebd.). Es handelt sich im Kern um einen Videoplayer, der zahlreiche Annotationsmöglichkeiten bietet, durch die das Datenmaterial mit visuellen Analysekommentaren im Bild versehen, kodiert und (rudimentär) transkribiert werden kann. Im Laufe der Durchsicht des Materials konnte ich so einzelne Sequenzen in den Daten markieren, verschlagworten und unter Kategorien subsummieren. Dieses Vorgehen ist vor allem vor dem Hintergrund der videographischen Datenintensität von großer Bedeutung und hilft dabei, das Datenmaterial gleichsam , aufzubrechen " und die für die Analyse relevanten Aspekte im Sinne einer theoretischen Auswahl herauszukristallisieren. Die Güte meiner Interpretation ließ sich dabei dadurch effektiv steigern, dass die gemeinsame Datensitzung (Tuma, Schnettler und Knoblauch 2013, 87) fester Bestandteil der Videographie ist: So konnten Dritte zur Analyse hinzugezogen und in gemeinsamen Datensitzungen die von uns erhobenen, audio-visuellen Daten, in den verschiedenen Aufbereitungsformen durch Dartfish, deren Granulation kennzeichnend für die Phasen des Analyseprozesses ist (Rohmaterial, Bild-im-Bild-Schnitte, Vortrags- oder Sequenz-weise), mit anderen Soziolog/innen betrachtet und diskutiert werden.Das ermöglichte, neben der solitären Analyse und der Analyse in der Projektgruppe, auch gemeinsam mit anderen, relevante Sequenzen zu finden oder Hypothesen über deren Bedeutung zu entwickeln, zu überprüfen und/oder zu verwerfen. Außerdem haben wir die Daten auch mit Feldteilnehmer/-innen betrachtet (Elizitationsinterviews (Knoblauch 2004a, Schubert 2008)).

Im Rahmen der audio-visuellen Analysen habe ich die konversationsanalytische Sequenzanalyse nach Harvey Sacks et al. (Sacks, Schegloff und Jefferson 1974) (Abschnitt 3.1.4), die erstmals durch Charles Goodwin (1979, 1981) methodologisch mit der Videoaufzeichnung verknüpft wurde sowie die VideoInteraktionsanalyse (im Folgenden VIA) (Tuma, Schnettler und Knoblauch 2013) als etablierte Auswertungsverfahren audio-visueller Daten angewendet. Beide Verfahren sind methodologisch in der Videographie verankert. Zusätzlich bediente ich mich der von Luckmann und anderen entwickelten GA (siehe 
Kapitel 3), um die videographierte Situation mittels meines ethnographischen Wissens sozialstrukturell zu kontextualisieren (Kapitel 4). Die genannten Verfahren der Konversationsanalyse (im Folgenden CA), VIA und GA greifen dabei ineinander: Sie ermöglichen es, die aufgezeichneten Situationen übersituativ zu verknüpften. So ist es z. B. möglich, typische Turn-Taking-Sequenzen (CA) in spezifischen Interaktionssituationen (VIA) zu erkennen, zu analysieren und als institutionalisierte, kommunikative Lösungen für wiederkehrende Problemlagen bzw. als entweder wenig(er) verfestigte kommunikative Formen oder als stark verfestigte kommunikative Gattungen (GA) zu bestimmen.

Entsprechend den von mir (auf Grundlage theoretischer Vorannahmen (Kapitel 2)) angenommenen maßgeblichen Einflussfaktoren auf die fokale Situation, den Group-Talk, nämlich: struktureller Kontext, Interaktions- und Kommunikationsweise sowie den drei korrespondierenden Analyseebenen der GA (Abschnitt 3.2), ziehen meine audio-visuellen Analysen drei analytisch zu trennende Ebenen in Betracht: Erstens spielt, für die Interaktion sowie die Kommunikation $^{11}$ im Group-Talk, das räumliche Setting und die technische Infrastruktur eine hervorgehobene Rolle. Entsprechend ist die VIA auf detaillierte Daten bzgl. der räumlichen Anordnung von Interaktionsteilnehmer/-innen und Objekten in der sozialen Situation angewiesen, wie sie die audio-visuellen Daten aus der Videographie bereitstellen. Ebenso essenziell sind die Körper-und Gesichtsformationen (Knoblauch 2007, 119, Kendon 1990, 210ff) der interagierenden bzw. kommunizierenden Teilnehmer/-innen innerhalb einer sozialen Situation für die VIA. Erst durch die Berücksichtigung der körperlichen Aspekte der Kommunikation wird es möglich, genauer zu bestimmen, wer eigentlich mit wem wie interagiert. Für diese verkörperten Wechselwirkungen spielen dann das räumliche Setting und die technische Infrastruktur ihre hervorgehobene Rolle. Wer blickt wen an, worauf lenken die Teilnehmer/-innen ihren körperlichen Aufmerksamkeitsfokus, welche technische Infrastruktur kommt dabei in welchem Moment zum Einsatz etc.

Zweitens liefert die CA Aufschluss über die lokale Organisation von Redezugwechseln, also über das Wie der sequenziellen Abfolge von ,turn taking[s]“ (Sacks, Schegloff und Jefferson 1974). Gerade in weniger formalisierten Gesprächssituationen (wie exemplarisch in der Konversation) ist der Redezugwechsel situativ organisiert. Wer wann das Wort erhält - oder sich selbst zur nächsten Redner/-in bestimmt - wird in der Situation ausgehandelt. Die Analyse

\footnotetext{
${ }^{11}$ Im Sinne eines weiten Kommunikationsbegriffs fallen Interaktion und Kommunikation in eins. Aus analytischen Gründen soll hier aber auf die unterschiedlichen Datensorten bzw. Analyseverfahren aufmerksam gemacht werden, die (sichtbare) nonverbale (VIA) von (hörbarer) (para-)verbaler (CA) Kommunikation unterscheiden.
} 
der Redezugwechsel gibt daher Aufschluss darüber, wer sich in einer Gesprächssituation als legitime Sprecher/-in begreift bzw. diese Rolle in Interaktion mit anderen behaupten kann. Die CA erlaubt darüber hinaus, die Analyse der thematischen Fokussierung im Rahmen von informellen Gesprächen, deren Thema nicht vorab festgelegt ist, sondern situativ bestimmt wird. Schließlich ermöglicht die CA auch eine Festlegung bzgl. der Analyseeinheit. Die Frage nach der kleinsten Einheit einer Analyse ist durchaus berechtigt: Soll man Silbe für Silbe, Wort für Wort oder Satz für Satz bzw. mit Bezug auf das visuelle Datenmaterial: Bild für Bild, Minute für Minute oder Video für Video analysieren? Welche Einheit kann im Sinn eines verstehenden Ansatzes (siehe Kapitel 2) als angemessen betrachtet werden?

Während diese Fragen, in verschiedenen visuellen Ansätzen der Soziologie, unterschiedlich beantwortet werden (Wilke 2020), bildet in der Videographie die Sequenz, d. h. die abgeschlossene Sinneinheit aus Perspektive der Feldteilnehmer/innen, die analytische Grundeinheit. Diese zu bestimmen, erlaubt uns die ethnomethodologisch informierte CA, in dem sie aufzeigt, dass Sprechende sich in ihrer Kommunikation selbst stets wechselseitig anzeigen, wann eine Sequenz beginnt, endet, unterbrochen und wieder aufgenommen wird etc. Entsprechend dieser Ethnomethode bzw. ihrer analytischen Nutzbarmachung durch die CA wurden die Sequenzen für die vorliegende Untersuchung bestimmt.

Drittens ermöglicht das ethnographische Wissen, das durch die teilnehmende Beobachtung (inklusive des Dokumentenstudiums) sowie die Expert/inneninterviews gewonnen wurde, die Forschungsresiduen aus der VIA sowie der CA zu explizieren. Dabei werden die Kenntnisse aus Beobachtung und Expert/-inneninterviews zur Interpretation der sozialen Situation hinzugezogen. Das ethnographische Wissen erschließt sich aus dem Forschungsfeld selbst, wobei es dieses zugleich elizitiert und den Forscher/-innen das Wissen über den institutionellen Rahmen zur Verfügung stellt, in den jede soziale Situation stets eingebunden ist. So wurden z. B. die disziplinären Hintergründe der einzelnen Teilnehmer/-innen am Group-Talk, insbesondere der Sprecher/innen, in Interviews erhoben, ohne die eine entsprechende Zuordnung, allein aus den beobachteten und videographierten sozialen Situationen, unmöglich gewesen wäre. Gerade aber in der disziplinären Heterogenität lag das konstitutive Moment der Situation überhaupt begründet, sodass sich an diesem Beispiel der Interview-basierten Erhebung der unterschiedlichen formalen Ausbildung der verschiedenen Wissenschaftler/-innen innerhalb der Forschungsgruppe klar verdeutlichen lässt, dass audio-visuelle Daten einer elizitierenden Ethnographie bedürfen, um analytisch einer Videographie zu genügen. 
Hier zeigt sich, dass audio-visuelle Daten die ,Wirklichkeit" niemals in ihrer ganzen, relevanten Phänomenbreite ,konservieren' können. Einerseits müssen sich Videograph/-innen bewusst sein, dass die Videoaufzeichnung stets nur einen Ausschnitt repräsentiert (Schnettler 2001, 143), weshalb die Ausrichtung der videographischen Kamera nicht zufällig sein darf, um den Anforderungen einer Videographie zu genügen. Andererseits geht aus dieser erkenntnistheoretischen Einschränkung deutlich hervor, dass Vorwissen, Erfahrung und die Erhebung von Feldwissen im Rahmen von Expert/-inneninterviews (Gläser und Laudel 2010), mit einem Wort: Ethnographie, unbedingt notwendig ist, um den Kamerablick einer Videographie anzuleiten. Umgekehrt bedeutet dies, dass die Methode keineswegs beliebig ist und nicht jede Analyse eines audio-visuellen Datums eine Videographie genannt werden kann.

Voraussetzung der Videographie ist die methodische Erhebung des audiovisuellen Datums durch Wissenschaftler/-innen, die über ethnographisches Kontextwissen verfügen (Knoblauch und Wilke 2018). Videographie ist daher methodologisch in die Fokussierte Ethnographie eingebettet, die ihrerseits nicht allein auf Videoaufzeichnung basiert, sondern, wie oben detailliert erläutert, auf einer für viele qualitative Forschungsdesigns typischen, ethnographisch informierten Methodentriangulation.

\subsubsection{Expert/-inneninterviews}

Bei den geführten Interviews handelt es sich, wie bereits kurz angesprochen, neben zwei Elizitationsinterviews, um qualitative Expert/-inneninterviews, die wir sowohl mit Teilnehmer/-innen der beobachteten Forschungsgruppe (EXP_1-5, EXP_11) als auch mit weiteren Expert/-innen im Feld (Kooperationspartner/innen der CNS/der konkreten Forschungsgruppe) (EXP_6-10) geführt haben. Gemäß unseres Erkenntnisinteresses hatten wir vorgängig einen Gesprächsleitfaden entwickelt (siehe unten: Tabelle 1.1), von dem wir allerdings spontan abwichen, wenn sich die Gesprächspartner/-in für die vorgesehenen Fragen als weniger ergiebige Informant/-in oder aber ein anderes Thema sich kontextuell als relevanter herausstellte. ${ }^{12}$

Die Gesprächspartner/-innen wählten wir nach Gesichtspunkten des Theoretical Sampling (Glaser und Strauss 2005[1967]) aus. Diese, dem Prinzip der Induktion folgende qualitative Datenerhebungsstrategie innerhalb der empirischen Sozialforschung beschreibt den Prozess, innerhalb dessen sich Verstehen

\footnotetext{
${ }^{12}$ Weitere Anpassungen waren notwendig, als wir später Interviews mit Expert/-innen führten, die selbst keine CNS-Forscher/-innen waren, aber mit dem Feld der CNS in Kooperationsbeziehungen standen (EXP 6-10).
} 
auf Grundlage zuvor erhobener empirischer Daten ergibt (ebd., S. 12). Im Verlauf dieses Prozesses entwickelt sich die Erkenntnis folglich inkrementell, durch den fortlaufenden Wechsel aus Datenerhebung und Auswertung. Er ähnelt dabei dem Vorgehen der Sozialwissenschaftlichen Hermeneutik (hermeneutischer Zirkel), wo sich das Vorwissen der Interpret/-innen durch den immer neuen Blick auf den Auslegungsgegenstand allmählich zu einem höheren Verstehen entwickelt. In Bezug auf die Auswahl unserer Gesprächspartner/-innen spielte zusätzlich das sog. Schneeball-Prinzip eine wichtige Rolle, wonach man, dem Credo des Theoretical Samplings entsprechend, neue Informant/-innen durch vorangegangene gewinnt. So waren es z. B. kurze Konversationen vor, während oder nach unseren Interviews, durch die wir von weiteren Personen erfuhren, die als Gesprächspartner/-innen für unsere Fragestellungen in Betracht kamen. Dies gilt insbesondere für die Interviews, die wir im Rahmen der beobachteten Forschungsgruppe führten (EXP_1-5, EXP_11).

In der Gesprächsführung achteten wir darauf, eine an die alltägliche Sprechweise der Befragten angepasste Rede zu pflegen. Dies beinhaltete insbesondere die Vermeidung von soziologischen Fachtermini und des Eindrucks, die Befragten selbst würden durch uns in irgendeiner Form (sozialwissenschaftlich) evaluiert werden. Eventuell motiviert durch entsprechende Befürchtungen, drückte eine Interviewpartnerin aus der Forschungsgruppe tatsächlich explizit Sorge aus, wir könnten Informationen aus dem Interview mit ihr weitergeben (EXP_3).

Wir haben diese Verunsicherung seitens der Beforschten, nicht zuletzt im Kontext weiterer Aussagen während des Gesprächs sowie auf Grundlage unserer allgemeinen Erfahrung innerhalb unseres Forschungsfelds, zum Teil einer unvollständigen Aufklärung der Gruppe durch den Forschungsgruppenleiter zugeschrieben. Dieser hatte uns zwar gestattete, uns mit einem eigenen Vortrag im Group-Talk vorzustellen und unser Forschungsinteresse zu explizieren. Jedoch hatte er weder im Vorfeld noch später selbst unser Dasein im Kontext der Gruppe erläutert. Zum anderen erklärten wir uns die geäußerte Befürchtung auch durch das stark konkurrenzierende Verhältnis der Gruppenmitglieder untereinander, das sich auch im Group-Talk beobachten ließ und dazu führte, dass unser Blick hinter die Kulissen der Einzelnen zum Teil von Skepsis begleitet wurde. ${ }^{13}$

\footnotetext{
${ }^{13}$ Später mussten wir feststellen, dass nicht nur die Teilnahme an bzw. die Offenheit in den Interviews, sondern auch die methodologisch für unser Vorhaben so zentrale Videoaufzeichnung im Group-Talk als Risiko für die wissenschaftliche Reputation bzw. die Hoheit über das vorgetragene geistige Eigentum begriffen wurde. Der Zugang stellte für uns somit, neben der Heterogenität und Spezifizität der im Feld diskutierten Wissensbestände, die größte praktische Herausforderung im Feld dar. Entsprechend resümieren wir, in Bezug auf diese Schwierigkeiten, in dem das Projekt beschließenden Forschungsbericht:
} 
Tabelle 1.1 Interviewleitfaden

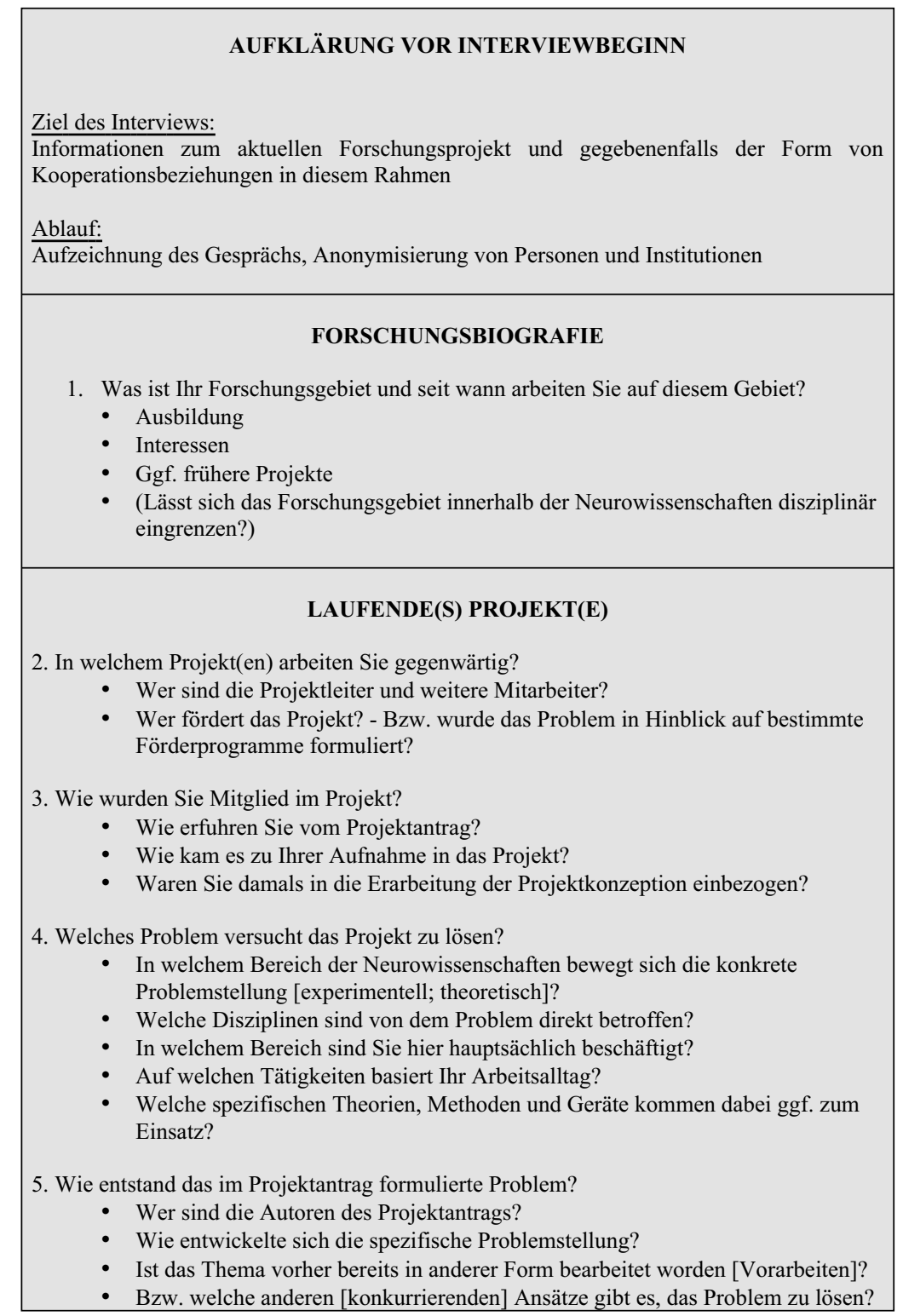

(Fortsetzung) 
Tabelle 1.1 (Fortsetzung)

\section{KOOPERATION UND KOMMUNIKATION}

6. Gibt es Kooperationsbeziehungen in Bezug auf ihre Arbeit?

[Falls , Nein', weiter zu 14.]

- Wer sind ggf. die Kooperationspartner?

- Aus welchen Fachgebieten stammen die Kooperationspartner?

- Sind die Ergebnisse ihrer Arbeit Voraussetzung für die Arbeit der Partner?

- Benötigen Sie die Leistungen anderer Kooperationspartner? Welche?

- Gibt es ein übergreifendes Problem, an dessen Lösung Sie und die Kooperationspartner arbeiten?

- Worin besteht die Zusammenarbeit ganz konkret?

7. Worüber tauschen Sie sich mit den Kooperationspartnern aus?

- inhaltliche Sachverhalte (Problemstellungen, technische Lösungsansätze)

- Personal-, Technik- und Ressourcenfragen (Raumnutzung, Gerätenutzung, Anforderungen an Geräte, Verteilung finanzieller Mittel)

- Konfligierende Ansichten

8. Welche Gelegenheiten existieren, um sich mit Kooperationspartnern auszutauschen?

- Gibt es regelmäßige Arbeitstreffen (z.B. Kolloquien, Konferenzen oder auch informellere Gelegenheiten)?

- Gibt es spezifische Kommunikationswege (z.B. Softwaretools, Videokonferenzen)?

- Wie oft nutzen Sie diese Gelegenheiten?

9. Gibt es eine gemeinsame „Sprache“?

[Falls, Nein', weiter zu 12!]

- Brückenkonzepte (Theorien, Methoden, Metaphern, materielle Modelle)

- Metasprachen (Mathematik, Formalismen)

- Visuelle Repräsentationen

- Häufigkeit und Art von Missverständnissen

10. Wie schätzen Sie die Funktionalität dieser „Kommunikationsformen“ einerseits für die konkrete Kooperation und andererseits für das eigene Forschungsfeld im Allgemeinen ein?

11. Wurden diese Kommunikationsformen [Brückenkonzepte, Metasprachen, Repräsentationen etc.] neu entwickelt oder bestanden diese [z.T.] bereits vorher?

- Steht der zeitliche Aufwand für die Kommunikation in einem positiven Verhältnis zum Output der Kooperation oder ist sie der eigenen Produktivität eher abträglich?

12. In welchen Form(en) werden (Zwischen-)Ergebnisse der Kooperation aufbereitet und dargestellt?

- Berichte

- Bilder

- Poster

- Konferenzbeiträge

- Gemeinsame Artikel in Fachzeitschriften

13. Werden Sie die aktuellen Kooperationen in zukünftigen Projekten fortsetzen? 
Tabelle 1.1 (Fortsetzung)

\section{ZUKÜNFTIGE PROJEKTE BZW. PROSPEKTION}

14. Planen Sie in Ihrem gegenwärtigen Forschungsbereich Anschlussprojekte bzw. in welchen anderen Forschungsbereichen möchten Sie in Zukunft arbeiten?

15. Welche mittel- und langfristigen Entwicklungen, die sich aus dem heutigen Stand evtl. bereits einschätzen lassen, erwarten Sie auf Ihrem Forschungsgebiet in den kommenden Jahren bzw. Jahrzehnten?

16. Offenheit für Nachfragen und zukünftige Interviews?

Vielen Dank für Ihre Zeit!

Im Rahmen der Interviews erlangten wir das grundlegende Vertrauen ausgewählter Gruppenmitglieder, die uns, ohne vorangegangenes Interview, die Erlaubnis, ihren Group-Talk zu filmen, nicht erteilt haben würden. Inhaltlich erfuhren wir wesentliches über die Professionen, Forschungsgebiete und konkreten Forschungsprojekte, in denen unsere Informant/-innen und andere Gruppenmitglieder sich (derzeit) engagierten. Neben den Forschungsbiografien lag der zweite Schwerpunkt der Interviews auf dem Bereich Kooperation und Kommunikation. In diesen Teilen erzählten uns unsere Gesprächspartner/-innen einerseits, wo vorhanden, von externen Kooperationspartner/-innen und andererseits von der Kooperation innerhalb der Gruppe selbst. Außerdem gewährten sie

Neben generellen Bedenken in Bezug auf die mögliche Verletzung ihrer geistigen Eigentumsrechte, spielten, wie wir später feststellten, vor allem der interdisziplinäre Kontext der Forschungsgruppe sowie der spezielle Werkstattcharakter des wöchentlichen Kommunikationsformats (Group Talk) hierbei eine wichtige Rolle. Zudem waren viele Teilnehmer/-innen mit unserem qualitativen Vorgehen nicht vertraut. Schließlich befürchteten viele der For-scher/-innen im Feld, dass unvollständige Forschungsergebnisse durch uns nach außen kommuniziert werden könnten. Trotz des prinzipiellen Einverständnisses des Forschungsgruppenleiters, erwies es sich daher zunächst als schwierig, die Erlaubnis zur Videografie [...] einzelner Sitzungen der Forschungsgruppentreffen zu erhalten. Da die Videografie einen wesentlichen Pfeiler unseres qualitativen Forschungsdesigns darstellte, wendeten wir viel Geduld und beharrliche Bemühungen dafür auf, die Erlaubnis von einigen der Wissenschaftler/innen trotzdem zu erhalten. Durch Beharrlichkeit gelang es uns auch, nicht zuletzt durch die geführten Expert/-innengespräche sowie durch die regelmäßige Teilnahme an den Treffen der Gruppe, die Zweifel bezüglich unserer qualitativen Methoden und der Anonymisierung des Datenmaterials auszuräumen und das Vertrauen von genügend Mitgliedern der Forschungsgruppe zu erlangen. (Knoblauch, Wilke und Lettkemann 2017, 3) 
uns hier Einblicke in die Bedeutung der Kommunikation und von Visualisierungen im Prozess dieser Kommunikation, sowohl innerhalb der eigenen Gruppe als auch im weiteren Kontext der CNS-Community. Zusätzlich erwähnenswert sind sicherlich die Informationen über die CNS im Allgemeinen, die Forschungsgruppe und die Arbeit verschiedener Forschungsgruppenmitglieder, nach denen wir nicht gefragt hatten und die unsere Informant/-innen zum Teil beiläufig oder unerwartet mit uns teilten, sodass die Interviews unsere Kenntnisse vom Feld auch in überraschender Weise bereichert haben.

Die Auswertung der Interviews mittels MAXQDA orientierte sich an der Qualitativen Inhaltsanalyse nach Mayring (2010), ohne dass wir dessen Verfahren allerdings gänzlich ausschöpfen wollten. ${ }^{14}$ Wir bedienten uns, nach dem Lesen und der ersten Diskussion der Transkripte, insbesondere der zusammenfassenden, qualitativ-inhaltsanalytischen Kategorienbildung, wobei uns der vorentworfene Leitfaden der Interviews auch hier als Richtschnur diente. Auf diese Weise fanden wir die in Bezug auf unsere Forschungsfragen relevanten Aussagen der Gesprächsteilnehmer/-innen in den Transkripten, die wir hierauf paraphrasierten und analytisch reduzierten, um Kategorien zu bilden. Die bei Mayring vorgesehene, enge und weite Kontextanalyse, zur Explikation der Inhalte unserer Interviews, konnten auf Grundlage des ethnographischen Wissens sowie vor dem Hintergrund der angestellten GA auf ein Mindestmaß beschränkt werden, das in erster Linie dem Prinzip der Intercoderreliabilität Rechnung trug. Andererseits dienten uns, wie bereits erläutert, die zusätzlichen, die teilnehmende Beobachtung ergänzenden Informationen aus den Interviews dazu, uns die tatsächliche soziale Situation in der Forschungsgruppe bzw. konkreter im Group-Talk en Details zu explizieren.

Das Elizitationsinterview (Knoblauch 2004a, Schubert 2008) ist ein methodisch sehr anspruchsvolles Erhebungsinstrument, dessen wir uns zweimal bedienten. Es stellt eine Form des offenen Interviews dar, in dem die oder der Interviewte dazu gebracht werden soll, relevante Aspekte des beforschten Phänomens, für das er oder sie als Feldteilnehmer/-in eingeladen wurde, zu elizitieren. $\mathrm{Zu}$ diesem Zweck nutzten wir unsere Videoaufzeichnungen aus den Group-Talks als Stimuli, die es uns erlaubten, ganz konkret nachzufragen, was mit einem bestimmten Wort oder Satz gesagt, was mittels einer bestimmten nonverbalen

\footnotetext{
${ }^{14}$ Ich habe sämtliche Interviews gemeinsam mit meinem Kollegen Eric Lettkemann geführt. Einen Teil der Interviews habe ich zudem selbst transkribiert. Die übrigen Transkriptionen wurden von unseren studentischen Hilfskräften im Projekt erstellt: Mein Dank gilt hier Jakob Gerber, Julia Rothenburg und Arne Janz. Letzteren danke ich zudem für ihre Mitarbeit an der qualitativen Inhaltsanalyse mittels MAXQDA.
} 
Geste, einer Ziffer an der Tafel oder einer Visualisierung auf der Digitalfolie ausgedrückt oder bezweckt werden sollte. Auf diese Weise haben wir die Methode genutzt, um uns in einem Fall, von einem externen Experten des Felds, der mit der beforschten Gruppe überdies persönlich vertraut war, einen Beitrag aus dem Group-Talk detailliert erläutern zu lassen. Dies war insbesondere deshalb sehr hilfreich, weil gegenüber dem externen Experten unsere Scheu geringer war, Wissenslücken zu offenbaren und ganz unbekümmert nachzufragen, was wir nicht verstanden. Diese Elizitation fand bereits ganz am Anfang und anhand unseres ersten aufgezeichneten Group-Talks statt. In einem zweiten Fall haben wir die Methode genutzt, um gemeinsam mit einem Mitglied der Forschungsgruppe dessen Video-aufgezeichneten Group-Talk nochmals zu betrachten und so gezielt Fragen an den Experten (hier im doppelten Sinn) stellen zu können. Auf diese Weise gelang es uns, unser Vorverständnis seines Vortrags zu validieren, nachzuhaken und Lücken in Detailfragen zu klären, die auch nach wiederholter Analyse des entsprechenden Beitrags bei uns offengeblieben waren. In diesem zweiten Fall haben wir das Video-Elizitationsinterview selbst Video-aufgezeichnet und anschließend vollständig transkribiert. Sämtliche Interviews wurden von uns im Sinne orthographischer Richtigkeit transkribiert. Dies entsprach unserem, mit der Datensorte verbundenen Erkenntnisinteresse, dass sich - fern von linguistischen oder emotionssoziologischen Fragestellungen - allein auf das feldbezogene Expert/-innen-Wissen der Interviewten bezog. Entsprechend wurden Transkriptionskommentare nur sparsam eingesetzt.

\subsubsection{Teilnehmende Beobachtung}

Eine bedeutende Rolle für das (fokussiert) ethnographische Forschungsdesign dieser Arbeit kommt, wie bereits angesprochen, neben der Erhebung audiovisueller Daten, der persönlichen, räumlichen und zeitlichen Kopräsenz in der fokalen Situation selbst zu. Während uns die Interviews sowie das Dokumentenstudium vor allem mit Informationen über die interne und externe soziale Strukturierung der Forschungsgruppe und des weiteren CNS-Felds versorgten und die Videographie es uns ermöglichte, die Interaktions- und Kommunikationsprozesse der Forschungsgruppe im Group-Talk detailliert zu besehen und zu analysieren, erfuhren wir durch die beobachtende Teilnahme vor Ort, wie es ist, d. h. auch, wie es sich konkret anfühlt, mit den anderen der Gruppe und dem Forschungsgruppenleiter zusammen zu sein: Welche Wege der informellen Kommunikation gibt es am Fachgebiet, an dem die Forschungsgruppe beheimatet ist, welche gemeinsamen Rituale? Wie funktioniert die tagtägliche Zusammenarbeit und schließlich wie ist die Stimmung in der Gruppe und wie und wann verändert sie sich? 
Dies war z. B. regelmäßig dann der Fall, wenn der wöchentliche Group-Talk anstand. Wir bemerkten schnell, dass in der restlichen Woche wenig untereinander kommuniziert wurde. Dann waren die Flure des Stockwerks des Universitätsgebäudes, an dem die Gruppe arbeitete, nahezu verwaist und bis auf die Sekretärin des Fachgebietsleiters fanden sich kaum Ansprechpartner/-innen. Am Morgen des Group-Talks hingegen wich diese Stille einer kurzfristigen, aufgeregten Betriebsamkeit: Innerhalb von wenigen Minuten strömten die Gruppenmitglieder, häufig weitgehend wortlos, was der frühen Stunde geschuldet gewesen sein mag, in dem kleinen Seminarraum zusammen, in dem das Treffen regelmäßig stattfand, nahmen ihre Plätze ein, insbesondere der Forschungsgruppenleiter stets denselben, und erwarteten den Beginn der Sitzung, wobei sie sich in der Regel mit ihren Smartphones ablenkten.

Nur in seltenen Ausnahmen fanden vorgängig oder anschließend informelle Gespräche zwischen den Gruppenmitgliedern statt. Im Laufe dieser ,Flur-' bzw. ,Teeküchengespräche " wurde der Group-Talk nachbesprochen und die Gelegenheit genutzt, Expert/-innen des vorangegangen besprochenen Themenfelds zu kontaktieren und spezifische Nachfragen zu stellen, die sich entweder vorgängig ergeben hatten oder direkt aus der Debatte des Group-Talks resultierten. Besonders interessant für mich ist aber, dass diese Gespräche selten waren und stets nur wenige Teilnehmer/-innen aufwiesen. Maßgeblicher erwies sich, was im GroupTalk besprochen worden war. Diese und weitere Beobachtungen verstetigte ich häufig bereits vor Ort schriftlich (auf einem kleinen Ipad) in Form von kleineren Feldnotizen, die ich später zu meinen Videoanalysen und zur Verfertigung von Publikationen zur Explikation heranziehen konnte.

\subsubsection{Datenintegration und (Re-)Kontextualisierung}

Durch die für qualitative Forschungsdesigns typische Datenheterogenität stehen viele qualitative Forscher/-innen schließlich vor dem gleichen Problem wie ich selbst. Sie müssen die unterschiedlichen, im Rahmen einer Feldphase erhobenen Datensorten (Interviews, prozessproduzierte Daten, audio-visuelle Daten, Beobachtungen) und ihre entsprechenden, im Verlauf je spezifischer Datenauswertungsverfahren (qualitative Inhaltsanalyse, CA, VIA) gewonnenen Forschungsergebnisse integrieren und re-kontextualisieren. Während zum Zweck der Erhebung spezifischer Eigenschaften bzw. Aspekte eines Phänomens, wie ich oben erläutert habe, unterschiedliche Methodenwerkzeuge zum Einsatz kommen müssen, beschreiben die so generierten Daten anschließend auch ausschließlich den jeweils fokussierten Aspekt, für den sie ausgewählt wurden und den sie angemessen zu repräsentieren erlauben. Vor diesem Hintergrund stellt sich daher anschließend stets die Frage, wie man mit der Heterogenität von so 
unterschiedlichen Datensorten wie Interviews und audio-visuelle Daten verfahren sollte.

Die GA, deren Gegenstandsadäquanz ich bereits einleitend erläutert habe, erwies sich auch in Hinblick auf die heterogenen Datensorten, die ich zu integrieren hatte, als hilfreich und angemessen. ${ }^{15}$ Sie diente in meinem Forschungsdesign als strukturierende Holistik, die es mir ermöglichte, die unterschiedlichen Datensorten (siehe unten: Abbildung 1.6) wieder zusammenzuführen und im Sinne der vorliegenden kommunikationssoziologischen Fragestellung auszuwerten. So erlaubte mir das wissenssoziologisch informierte Verfahren der GA, meinen empirischen Betrachtungsgegenstand, der zuvor, in Erhebung und Auswertung der erhobenen Forschungsdaten, in Teilaspekte aufgetrennt wurde, auf einer höheren Aggregatsstufe, als Begriff zweiter Ordnung, als kommunikative Gattung, soziologisch zu rekonstruieren (Kapitel 4 und 5). In Abbildung 1.6 werden die drei maßgeblichen Einflussfaktoren auf die fokale Situation (institutioneller Rahmen, Interaktion und Kommunikation) mit den jeweils entsprechenden Datenerhebungsverfahren der Fokussierten Ethnographie (Expert/-inneninterviews, teilnehmende Beobachtung und audio-visuelle Daten) sowie den drei Strukturebenen der GA (Außenstruktur, situative Realisierung, Binnenstruktur) visuell in Beziehung zueinander gesetzt. (Aufgrund der hervorgehobenen Bedeutung der GA für meine Arbeit ist ihr im Folgenden ein eigenes Kapitel gewidmet (Kapitel 3).)

\footnotetext{
${ }^{15}$ Dies überrascht nicht, wenn man bedenkt, was in Kapitel 2 und 3 dieser Arbeit noch deutlicher werden wird, dass ich mich mit meiner Forschung in einer Denk- und Forschungs,Schule“ verorten darf, die, ausgehend von Schütz Verstehender Soziologie, über den SoKo bis zur GA, der Fokussierten Ethnographie sowie der Videographie und dem KoKo, ein elaboriertes, eng verwobenes Theorie- und Methodenfeld umfasst.
} 


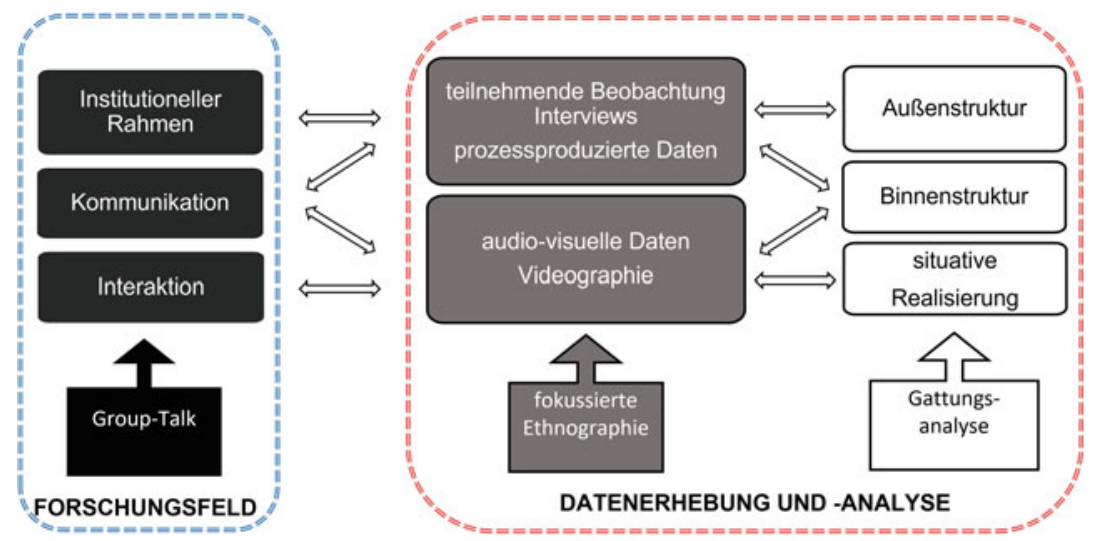

Abbildung 1.6 Schematische Darstellung meines Forschungsdesigns: von den Aspekten des empirischen Phänomens, über die Datensorten der Fokussierten Ethnographie (teilnehmende Beobachtung, Expert/inneninterviews, Videographie) zu den analytischen Ebenen der GA (Außenstruktur, situative Realisierung, Binnenstruktur)

\section{3 Überblick über die weiteren Kapitel der Arbeit}

Im folgenden Kapitel dieser Arbeit wende ich mich der theoretischen Verortung meiner empirischen Untersuchung zu (Kapitel 2). Hierzu unterscheide ich grundlegende Vorannahmen (verstehende Soziologie) und forschungsleitende Grundbegriffe (KoKo), die ich jeweils, aus der verstehenden Perspektive meiner Arbeit, ausführlich erläutern werde. Eine wichtige Rolle wird dabei auch die Auseinandersetzung mit dem meiner Arbeit zugrunde liegenden Akteurskonzept spielen, das, vor dem Hintergrund meiner spezifischen, kommunikationssoziologischen Fragestellung, von besonderer Bedeutung ist. Hierauf folgt, wie angekündigt, eine detaillierte Auseinandersetzung mit dem soziolinguistischen Konzept der Gattung, der sprach- (Luckmann) und kommunikationssoziologischen (Knoblauch) Gattungsanalyse sowie deren maßgeblicher Quellen (Kapitel 3). Das darauffolgende vierte Kapitel beinhaltet meine empirischen Analysen und stellt das eigentliche Herzstïck meiner Arbeit dar. Ausgehend von den Strukturebenen der GA werde ich dort, anhand der verschiedenen erhobenen Datensorten, das institutionelle Umfeld der Forschungsgruppe sowie ihre Kommunikations- und Interaktionsweise im Group-Talk detailliert darstellen, analysieren und in Hinblick auf meine im Verlauf der Forschungsarbeit gewonnene Hauptthese (siehe oben) auswerten (Kapitel 4). Im fünften und letzten Kapitel meiner Arbeit 
schließlich findet sich die Zusammenfassung meiner Forschungsergebnisse sowie das Fazit meiner Arbeit und ein Ausblick auf mögliche Fortführungen im Bereich der kommunikationssoziologischen Gattungsanalyse (Kapitel 5).

Open Access Dieses Kapitel wird unter der Creative Commons Namensnennung 4.0 International Lizenz (http://creativecommons.org/licenses/by/4.0/deed.de) veröffentlicht, welche die Nutzung, Vervielfältigung, Bearbeitung, Verbreitung und Wiedergabe in jeglichem Medium und Format erlaubt, sofern Sie den/die ursprünglichen Autor(en) und die Quelle ordnungsgemäß nennen, einen Link zur Creative Commons Lizenz beifügen und angeben, ob Änderungen vorgenommen wurden.

Die in diesem Kapitel enthaltenen Bilder und sonstiges Drittmaterial unterliegen ebenfalls der genannten Creative Commons Lizenz, sofern sich aus der Abbildungslegende nichts anderes ergibt. Sofern das betreffende Material nicht unter der genannten Creative Commons Lizenz steht und die betreffende Handlung nicht nach gesetzlichen Vorschriften erlaubt ist, ist für die oben aufgeführten Weiterverwendungen des Materials die Einwilligung des jeweiligen Rechteinhabers einzuholen.

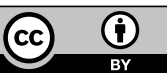

